\title{
Selection and experimental study of the linear displacement converter for the automated monitoring system of mine roofs
}

\author{
Maxim Inozemtsev ${ }^{1 *}$, Natalia Grigoreva ${ }^{1}$, Natalia Volynkina ${ }^{1}$, and Atabek Alibaev ${ }^{2}$ \\ ${ }^{1}$ T. F. Gorbachev Kuzbass State Technical University, Prokopievsk branch, The Department of \\ Information Technology, Engineering and Transport, 653039 Prokopievsk, 19a Nogradskaya str., \\ Russian Federation. \\ ${ }^{2}$ Institution Jalal-Abad State University named after Bekmamat Osmonov, 715600, Dzhalal-Abad, \\ Lenina 57, Kyrgyzstan.
}

\begin{abstract}
The justification of the linear displacement sensors installation in mine workings is described in this paper. The required technical characteristics of the sensors are given. The main types of primary linear displacement converters are presented. The choice of inductive solenoidtype converters and sensors based on linear differential transformers (LVDT) is justified. The advantages and disadvantages of inductive transducers, their accuracy and measurement limits are described, and measuring methods of inductance of the solenoid-type transducers and the converter output signal based on LVDT are considered. The results of sensors experimental studies based on the solenoid-type inductive transducers are presented.
\end{abstract}

\section{Introduction}

The increase in production volumes of ore and nonmetallic mineral extraction by underground method causes an increase in the intensity of the impact of mining equipment on the rock mass. Besides, the seams development, especially in decompacted or watered rocks, leads to the appearance of various geodynamic processes in mine workings. The decompression processes and roof collapses are the most typical. Unpredictable collapses capable of causing significant damage to machinery, support and equipment. For this reason, safety rules for mining operations provide monitoring the rock mass displacement. Mechanical sensors currently used to solve this problem do not allow to control with the required accuracy and sufficient promptness. This is due to the measurements low accuracy, the need of attracting staff to periodically take readings from sensors, visual readings and a high probability of error due to the human factor. In general, the problem of safe mining operations is urgent and is given great attention from both production staff and the scientific community. Mine safety is influenced by many factors, some of them are described in works [1 - 4]. The work [5] considers the creation of a system for measuring the stress-strain state of a rock mass based on the use of three-component strain gauges.

* Corresponding author: inozemcevma@kuzstu.ru 
A modern mining enterprise is complicated technological complex. Various automated systems and software complexes are increasingly used for production management. The task of automating the control of vertical displacements in a mine workings can be effectively solved by creating and integration automated monitoring systems described in [6]. The components of described system are linear displacement converters to a proportional electrical signal.

Displacement sensors should be installed in places prone to manifestation of various geodynamic processes, while it is necessary to ensure the measurement of displacements at three levels. The sensors are combined into a single network covering all mine workings, and information from each sensor will be sent to the dispatcher remote control and stored in the database for further analysis.

Based on the analysis it is possible to determine the technical requirements that the sensor in underground mine workings must meet:

- providing measurements at three levels;

- $\quad$ range of measured displacements from 0 to $100 \mathrm{~mm}$;

- $\quad$ supply voltage no more than $12 \mathrm{~V}$;

- type of output signal - digital;

- cylindrical body for ease of installation in mine workings.

In order to ensure compatibility of the sensor with industrial controllers and SCADA systems, data transfer capability in accordance with the EIA / TIA-485 (RS-485) standard or equivalent must be provided.

Currently, a large number of linear transducers are known. They are quiet differrent in physical principles of their operation, such as: resistive, capacitive, piezoelectric, inductive and other sensors. The type of transducer determines the possible field of application, the operating conditions and the range of the measured value. So, it is impossible to use some types of transducers in underground workings. For example, coal dust limits the use of resistive transducers because, being conductive, it can affect the resistance of the transducer, distorting the measurement results. The use of capacitive sensors is also complicated by the increased humidity. Thus, the use of inductive transducers seems to be the most optimal in underground mine working conditions, especially in coal mines, characterized by the presence of coal dust and high humidity.

\section{Theory}

The operating principle of inductive transducer is based on changing its inductance depending on the magnitude of the linear displacement. Structurally, inductive transducers can be performed in various ways, however, they contain one or more windings and, as a rule, a ferromagnetic core.

The main advantages of inductive transducers include: relative simplicity of design; the possibility of obtaining high metrological characteristics; high sensitivity; small enough size. Herewith, structurally inductive transducer can be made in a sealed housing and the measured displacement is applied only to the movable rod, thereby fulfilling the requirement to reduce the impact of the external environment.

The disadvantages of inductive transducers are the dependence of inductance on external magnetic fields. Therefore, it is unacceptable to place inductive transducers near sources of strong constant or alternating magnetic fields, which include powerful power equipment, as well as high voltage electrical cables or intended for powering powerful consumers. If it is necessary to place the transducers in such places, it is necessary to provide the converters shielding or use another method of measuring displacements.

There are great number of different inductive converter implementations. These include transducers with a variable air gap, with a variable area of the gap, transducers with an open 
magnetic circuit. Besides, single and differential (transformer) transducers are known. To solve the set problem, the most suitable are solenoid-type transducers, or transducers made on the basis of linear differential transformers.

\subsection{Inductive converters}

An inductive solenoid-type transducer is an inductance coil, along the axis of which a ferromagnetic movable core moves. The principle of inductive solenoid-type transducer operation is based on changing the inductance of the solenoid depending on the position of the moving core.

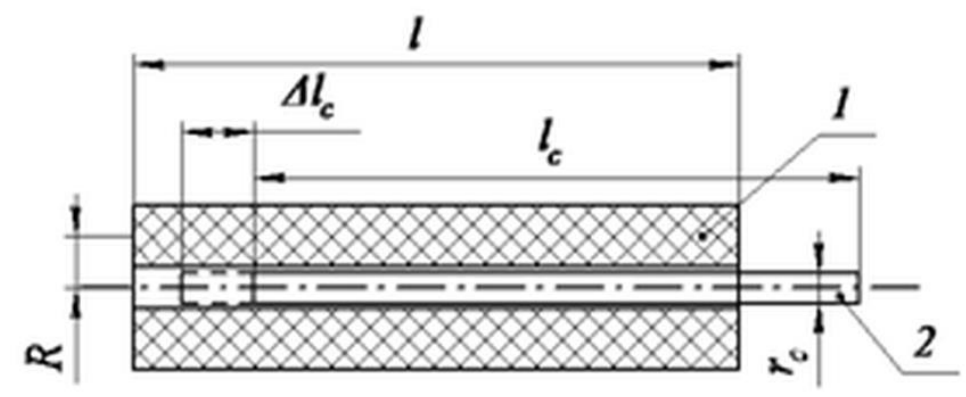

Fig. 1. The device is an solenoid-type inductive converter. 1 - winding; 2 - movable core.

The inductance of a solenoid-type transducer with a ferromagnetic core is determined by the expression [7]

$$
L=\frac{\pi \mu_{0} N^{2}}{l^{2}}\left[r^{2}\left(\sqrt{r^{2}+l^{2}}-r\right)+\left(\mu_{m}-1\right) r^{2}\left(\sqrt{r_{c}^{2}+l_{c}^{2}}-r_{c}\right)\right],
$$

where $\mu_{0}-$ is the magnetic constant;

$N$ - the number of turns of the winding;

$r$ - the radius of the coil of the transducer;

$l$ - length of the coil of the transducer;

$\mu_{m}$ - relative magnetic permeability of the core material;

$r_{c}$ - core radius;

$l_{c}-$ is the length of the core.

When the core is displaced by $\Delta l_{c}$, the change in inductance will be [7]

$$
\Delta L=\frac{\pi \mu_{0} N^{2}}{l^{2}}\left(\mu_{m}-1\right) r^{2} \frac{l_{c}}{\sqrt{r_{c}^{2}+l_{c}^{2}}} \Delta l_{c}
$$

From expressions (1) and (2) it can be seen, that with the known parameters of the transducer, the offset value can be determined by measuring the inductance of the transducer:

$$
\Delta l_{c}=\frac{\Delta L}{\frac{\pi \mu_{0} N^{2}}{l^{2}}\left(\mu_{m}-1\right) r^{2} \frac{l_{c}}{\sqrt{r_{c}^{2}+l_{c}^{2}}}}
$$

Inductance measurement can be performed in various ways. A common method is when the oscillation frequency of an $L C$-oscillator is measured, the circuit of which includes the measured inductance. The autogenerator can be made on the basis of any scheme, for example, the Kolpitz, Klapp and others generator. The bipolar and field-effect transistors, 
operational amplifiers, and logic elements can be the reinforcing elements. It is advisable to measure the oscillation frequency with a microcontroller. The advantage of this method is the availability of the element base. However, it becomes difficult to ensure stable operation of the generator and a constant level of the output signal amplitude in a wide range of inductance variation, which leads to the complication of the circuit.

The known method is based on measuring the duration of the transition process $R L$ circuit, determined by the time constant $\tau$, depending on the inductance of the converter and the total active resistance of the converter and resistor. The process duration is measured when the circuit is connected and disconnected from the power source, or by applying a sequence of square-wave pulses to the $R L$-circuit. The implementation of this method implies the use of a comparator. The computing device is also a microcontroller. The advantage of this method is the simplicity and a small number of electronic components required for the operation of the circuit. However, to ensure high accuracy, it is necessary to use precision elements, as well as highly stable reference voltage sources or square wave generators.

The bridge measurement method is highly accurate and is often used in laboratory conditions. Application of the method for solving the problem posed requires the presence of a reference inductance in the measuring circuit and a source of a sinusoidal signal with high requirements for frequency stability and output voltage level. However the high accuracy, the implementation of the measuring circuit is more complicated than in the previous versions.

Thus, when using an inductive converter, the most optimal for solving this problem is a circuit that measures the time constant of the $R L$-circuit.

\subsection{LVDT sensors}

Significantly greater opportunities for displacement measurements are provided by displacement sensors. The transducer in displacement sensors is a linear differential transformer (LVDT). Transformer-type sensors consist of a primary winding, and two secondary windings connected in opposite directions. Structurally, the windings are located on one axis. The core moves along this axis.

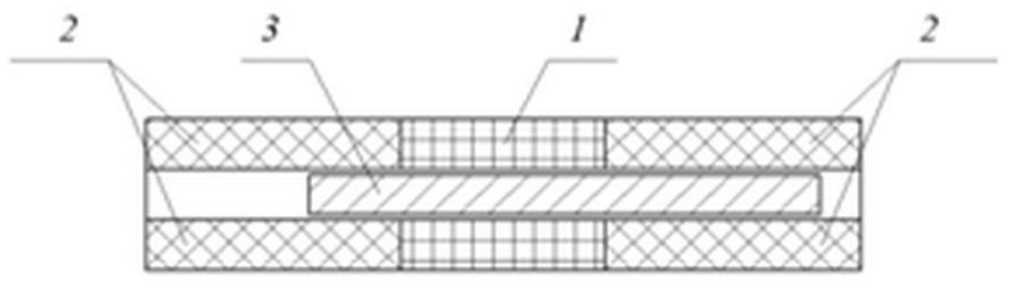

Fig. 2. Device of a linear differential transformer. 1 - primary winding; 2 - secondary windings; 3 core.

The operation principle of such a transducer is based on the change in the mutual inductances of the secondary windings of the transformer when the ferromagnetic core is moved (Fig. 3). A sinusoidal signal from a generator is applied to the primary winding, and the value of the output voltage and the phase of the signal received from the secondary windings depends on the position of the core. The advantage of LVDT sensors is the linearity of the output characteristic and high measurement accuracy, as well as the fact that these converters allow determining not only the amount of displacement, but also its direction.

Circuits that implement signal processing from LVDT sensor are, in general, more complicated than analogous ones for inductive solenoid-type sensors. This is due to the 
fact, that when developing measuring circuits based on LVDTs, it is necessary to measure not only the output voltage of the secondary windings, but also apply phase detection to obtain information about the bias direction. Taking into account the widespread use of such sensors in various fields of science and technology, specialized microcircuits for processing signals with LVDTs - AD697 and AD698 were developed.

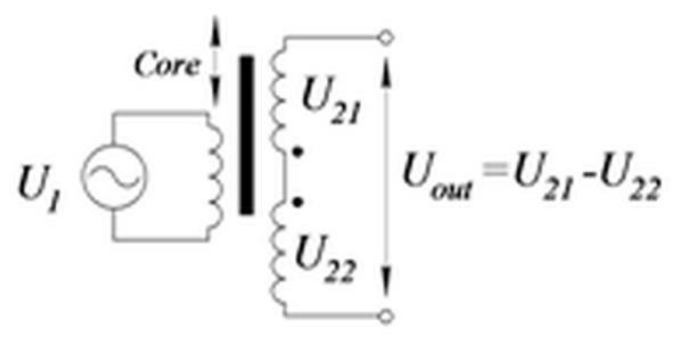

Fig. 3. Basic LVDT circuit.

LVDT - sensors are more widely used in comparison with solenoid sensors. This explains the significant amount of work performed on this type of sensor. Currently, researchers develop an output signal processing circuit [8 - 13] and LVDT designs [14 $15]$.

\section{Results and discussions}

A prototype of a displacement sensor with three transducers of the solenoid-type was experimentally investigated in this work. The transducer coil contains 2000 turns of wire with a diameter of $0.25 \mathrm{~mm}$, wound on a frame with a diameter of $7 \mathrm{~mm}$ and a length of $100 \mathrm{~mm}$. The inductance of such a coil without a ferromagnetic core is about $2.5 \mathrm{mH}$. The resistance of the coil wire is 19 ohms. Structurally, the sensor is a cylindrical body with inductive converters and a printed circuit board inside it.

To measure the inductance of the converter, a method based on measuring the time constant of the $R L$-circuit, consisting of a converter coil and a resistor of $51 \mathrm{Ohm}$ was chosen (Fig. 4). The computing device is a 32-bit microcontroller.
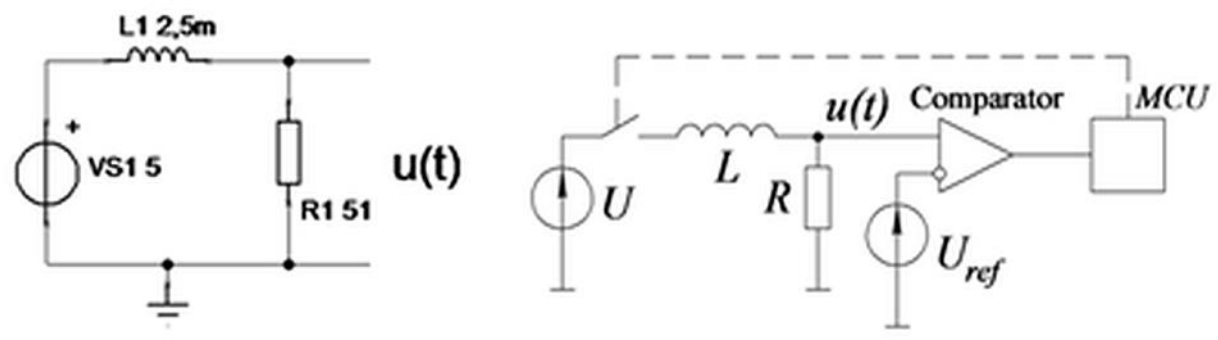

Fig. 4. Scheme for measuring the time constant of inductive converter and its simplest implementation.

When the $R L$-circuit is connected to a voltage source, the current in the circuit is determined by the expression

$$
i(t)=\frac{U}{r}\left[1-\exp \left(-\frac{t}{L} R\right)\right]
$$

The current $i(t)$, passing through the resistor $R$, creates a voltage drop across it equal to 


$$
u(t)=U\left[1-\exp \left(-\frac{t}{L} R\right)\right]
$$

Whence the inductance of the transducer is

$$
L=-t R \frac{1}{\ln \left[1-\frac{u(t)}{U}\right]}
$$

The inductance increment is equal to the difference between the current inductance value determined by expression (6) and the initial inductance value at a certain position of the core $L_{0}$

$$
\Delta L=L-L_{0}
$$

The voltage $v(t)$ is supplied to one of the inputs of the comparator (Fig. 4). While a reference voltage source of $V_{r e f}=3.3 \mathrm{~V}$ is connected to the other input of this comparator. After a time $t$ from the beginning of the transient process, the voltage across the resistor and at the comparator input, will become equal to the reference, and its output will set a high level, interpreted by the microcontroller as a logical high. A change the logic level at the microcontroller input leads to the formation of an interrupt that in accordance with the microcontroller operation algorithm stops the counter. Since the counter starts at the moment the voltage is applied to the $R L$-circuit, then microcontroller determines the time $t$ by reading the value of the counter. Knowing the time $t$ the inductance of the transducer is calculated from expression (5), and the amount of displacement is calculated by expression (3). The inductance calculation results of each converter were transmitted by the microcontroller via the UART interface to the computer.

In fig. 5 one can see a graph of the transient process of an $R L$-circuit, with an inductance $L=2.5 \mathrm{mH}$ and a total active resistance of $70 \mathrm{Ohm}$. The circuit was connected to a source with a voltage of $5 \mathrm{~V}$. As seen from the graph, the time from the moment the voltage is applied to the circuit to the moment the comparator operates at the $3.3 \mathrm{~V}$ level is about 85 $\mu \mathrm{s}$. The measurement of such a time interval for modern microcontrollers can be performed with high accuracy, therefore this method provides the determination of the inductance, and hence the displacement, with the required accuracy.

Next, the inductance was measured and the amount of displacement was calculated for various positions of the core inside the coil. In the used model, when the core was displaced from 0 to $100 \mathrm{~mm}$, the inductance of the converter varied in the range of $2-25 \mathrm{mH}$. According to the results of the experiment, the error in determining the displacement did not exceed $0.2-0.3 \mathrm{~mm}$, that meets the technical requirements for the sensor. 


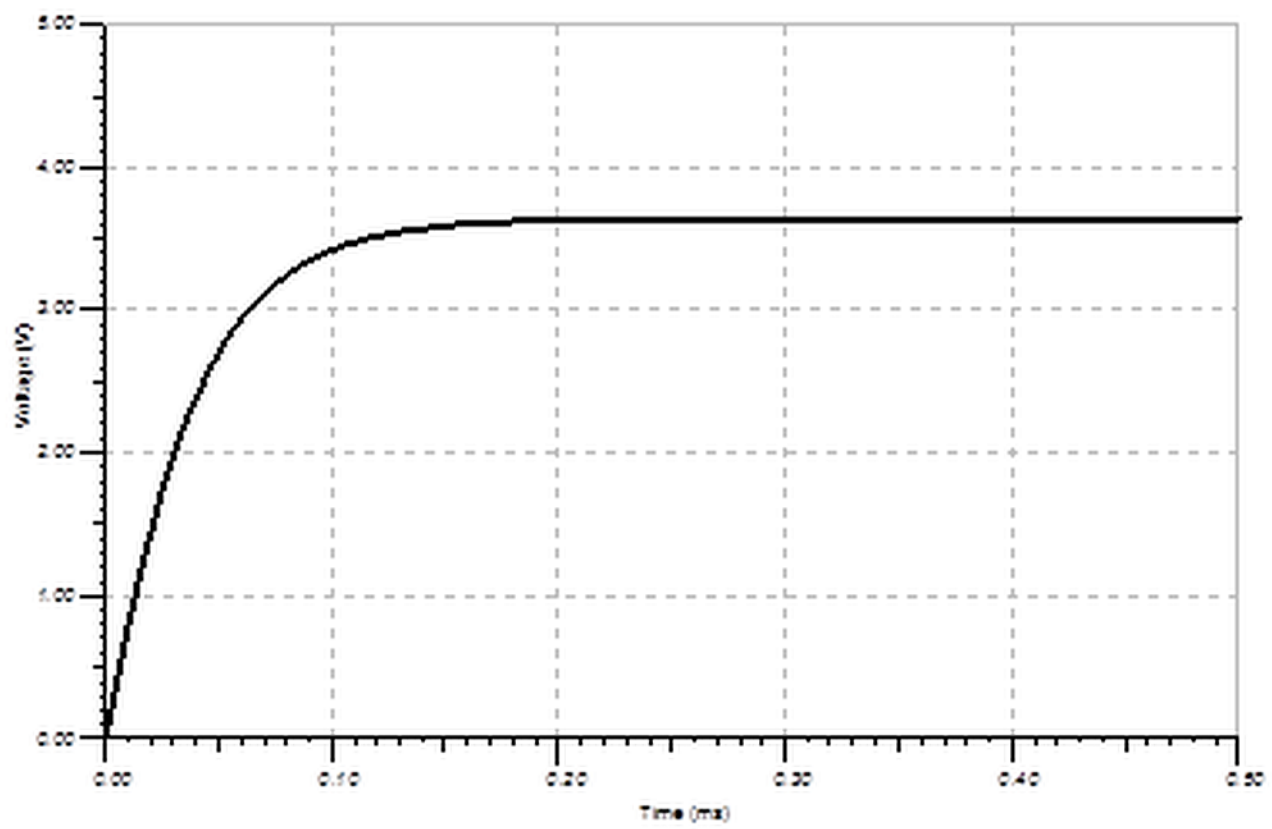

Fig. 5. Graph of the transient process of the converter experimental model (in the absence of a core).

Based on the review of the linear displacement transducers design and methods for measuring the output signals of converters, it was found, that when creating an automated system for monitoring the mine roof vertical displacements, it is advisable to use linear displacement inductive sensors. In this case, depending on the required accuracy and the need to determine the direction of the displacement, it is possible to use solenoid-type transducers, or transducers based on a linear differential transformer. Both types of transducers allow to perform a cylindrical sensor design, which simplifies the installation of sensors in underground mines. The implementation of an information system based on the developed sensors will automate the process of obtaining, storing and processing information about vertical displacements of the mine roof that will contribute to improving the safety of mining operations.

\section{References}

1. E Pudov, E Kuzin, A. Efremenkov, IOP Conf. Ser.: Mater. Sci. Eng., 253, 012063 (2020)

2. M. Mamaeva, E. Kuzin, MATEC Web of Conferences, 297, 03006 (2019)

3. B. Gerike, I. Panachev, E. Kuzin, E3S Web of Conferences, 15, 03008 (2017)

4. E. Kuzin, M. Mamaeva, B. Gerike, K. Singh, E3S Web Of Conf. IVth International Innovative Mining Symposium, 209, 03011 (2019)

5. K. Morozov, A. Shabarov, A. Kuranov, et al, E3S Web Conf., 129, 01012 (2019)

6. A. Abramovich, E. Pudov, E. Kuzin, E3S Web of Conf., 21, 01011 (2017)

7. Liu, Lvliang, IOP Conf. Ser.: Mater. Sci. Eng., 452, 042114 (2018) 
8. G. Vinodhini, S. Aniruddhan, B. George, J. D. Devi and P. V. Ramakrishna, Proceedings of the IEEE International Instrumentation and Measurement Technology Conference, I2MTC (2017)

9. H. Ganesan, B. George,S. Aniruddhan, Proceedings of the IEEE International Instrumentation and Measurement Technology Conference, I2MTC, 1 (2018)

10. W. Petchmaneelumka, P. Mano, V. Riewruja, Proceedings of the 2018 3rd International Conference on Control and Robotics Engineering ICCRE (2018)

11. W. Petchmaneelumka, K. Songsuwankit, A. Rerkratn, V. Riewruja, Proceedings of the 3rd International Conference on Control, Automation and Robotics, ICCAR (2017)

12. D. Sahu, S. Hazra, P. Nandi, Proceedings of the 29th International Conference on VLSI Design and 2016 15th International Conference on Embedded Systems (VLSID) (2016)

13. G. Chen, B. Zhang, P. Liu, H. Ding, IEEE Sens J, 15 (2015)

14. J. de Pelegrin, B. M. de Carvalho, F. Luiz Bertotti, J. S. Lafay, J. de Pelegrin, Proceedings of the 2nd International Symposium on Instrumentation Systems, Circuits and Transducers, INSCIT, 1 (2017)

15. N. Anandan, B. George, IEEE Sensors Journal, 17, 5298 (2017) 